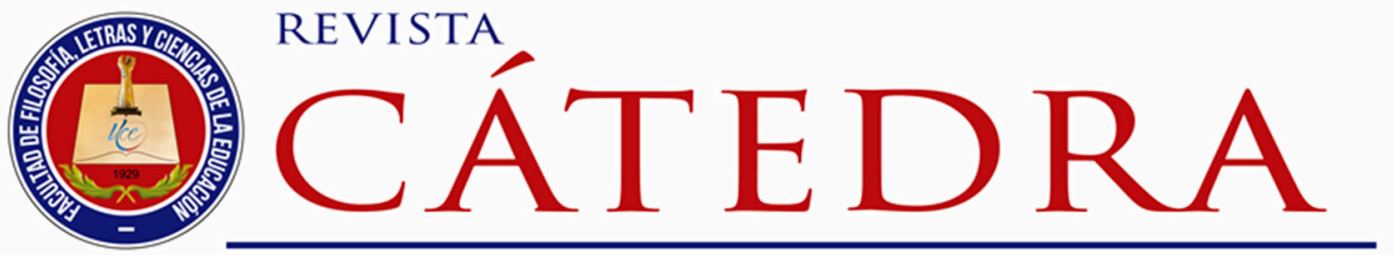

\title{
Las TIC en el contexto familiar en tiempos de confinamiento: experiencias de conectividad y comunicación de estudiantes universitarios
}

\author{
ICT in the family context in times of confinement: \\ connectivity and communication experiences of \\ university students
}

Jorge Silva-Castillo

Universidad Nacional de Chimborazo, Riobamba, Ecuador jsilva@unach.edu.ec https://orcid.org/0000-0001-8928-7201

Mirian Peñafiel-Rodríguez Universidad Nacional de Chimborazo, Riobamba, Ecuador mpenafiel@unach.edu.ec https://orcid.org/0000-0002-5327-1635

Amparo Cazorla-Basantes

Universidad Nacional de Chimborazo, Riobamba, Ecuador acazorla@unach.edu.ec https://orcid.org/0000-0003-0268-2722

(Recibido: 07/10/2021; Aceptado: 15/10/2021; Versión final recibida: 30/11/2021)

Cita del artículo: Silva-Castillo, J., Peñafiel-Rodríguez, M. y Cazorla-Basantes, A. (2022). Las TIC en el contexto familiar en tiempos de confinamiento: Experiencias de conectividad y comunicación de estudiantes universitarios. Revista Cátedra, 5(1), 47-57.

\section{Resumen}

Frente a la situación de emergencia sanitaria ocurrida desde el año 2020 por la pandemia mundial de coronavirus covid-19, la sociedad tuvo que irse adaptando a nuevos contextos de la mano de las Tecnologías de la Información y la Comunicación (TIC). El trabajo presencial se vio interrumpido en sus actividades cotidianas por el confinamiento obligatorio y a la par de aquello se optó por la modalidad virtual que durante varios años 
atrás ya se aplicaba en la educación superior a distancia y en ámbitos de la economía digital, pero ahora se vio fortalecida por la necesidad de seguir cumpliendo la misma labor específicamente en el campo educativo. La utilización de las TIC se ha convertido en un elemento imprescindible, y bajo esa necesidad confluyeron los actores sociales con distintas realidades, por lo cual el presente estudio tuvo como objetivo revelar cuales fueron las formas de acceso a la información y la comunicación por parte de los estudiantes de la Universidad Nacional de Chimborazo (UNACH) durante el año 2020. Se realizó un diagnóstico cuantitativo del porcentaje de conectividad, tipo de servicio, con un diseño no experimental transeccional descriptivo en referencia al acceso a dispositivos tecnológicos que tienen los estudiantes universitarios. Los resultados obtenidos en esta investigación permitieron conocer que un $70 \%$ de estudiantes de las cuatro facultades que conforman la UNACH durante el año 2020 hicieron uso compartido de dispositivos con otros miembros de la familia para ejecutar sus actividades académicas y de teletrabajo dentro del hogar.

\section{Palabras clave}

contexto, conectividad, confinamiento, covid-19, dispositivos, familiar, TIC

\section{Abstract}

Faced with the health emergency that has occurred since 2020 due to the global coronavirus pandemic (COVID-19), society had to adapt to new contexts with the help of Information and Communication Technologies (ICT). Face-to-face work was interrupted in its daily activities by the mandatory confinement and at the same time the virtual modality was chosen, which for several years had already been applied in distance higher education and in areas of the digital economy, but now it was strengthened by the need to continue fulfilling the same work specifically in the educational field. The use of ICT has become an essential element, and under this need social actors with different realities converged, so the present study aimed to reveal which were the forms of access to information and communication by students of the National University of Chimborazo (UNACH) during the year 2020. A quantitative diagnosis of the percentage of connectivity, type of service, with a descriptive non-experimental transactional design in reference to access to technological devices that university students have was performed. The results obtained in this investigation allowed to know that $70 \%$ of students of the 4 Faculties that conform the UNACH during the year 2020 made shared use of devices with other members of the family to execute their academic activities and telework within the home.

\section{Keywords}

Context, connectivity, confinement, covid19, devices, family, ICTs.

\section{Introducción}

La crisis sanitaria por el coronavirus (Covid-19) desde el mes de abril del 2020 convirtió a la tecnología en aquella alidada para continuar avanzando con las tareas del quehacer diario, siendo una alternativa necesaria para dinamizar servicios que en otra época hubiera significado una paralización parcial o total de las actividades. Es así que, a raíz de estas situaciones generadas por la pandemia, se ejecutó un proceso de indagación para mostrar a través de este trabajo investigativo de la situación de los estudiantes del nivel superior quienes desde diferentes sectores del Ecuador se vieron en la necesidad de dar continuidad a su desempeño académico (Chirinos et al., 2020).

La Universidad Nacional de Chimborazo (UNACH) desde su creación en el año 1995 ha impartido una educación de forma presencial y su currículo responde a ello, sin embargo, desde el año 2014 adoptó un modelo Blearning con su plataforma Moodle, es decir se fue 
adaptando a una modalidad combinada entre lo presencial y lo virtual, lo que permitió que las clases tanto síncronas como asíncronas con los estudiantes en el año 2020 se lleven a cabo bajo este contexto no tan desconocido. Sin embargo la presente investigación tiene su asidero en conocer sobre la realidad que experimentaron cada uno de los integrantes de una familia y como debían adaptarse a esta nueva modalidad por la misma necesidad de poseer equipamiento tecnológico, de conectividad, e incluso el conocimiento para el uso de estos servicios tecnológicos desde lo básico hasta lo más avanzado; a esto se suma factores como el compartir el mismo entorno de aprendizaje, la realidad económica dentro del hogar, entre otros.

Este trabajo toma relevancia a partir de esta situación de índole social que permitió conocer de qué manera se llevó a cabo la comunicación durante este tiempo de confinamiento entre los actores pertenecientes al seno familiar, del cual destacamos a los estudiantes universitarios y el uso que dieron a las herramientas y plataformas digitales en combinación con el denominado teletrabajo. El aporte de este estudio es pertinente no solo por el hecho de llevar a cabo una investigación por época de pandemia, sino porque se hizo notoria a partir de la obligatoriedad de ampararse en un modo virtual de conocer a fondo la situación de una sociedad que vive actualmente la Era Digital. No obstante, una nota periodística de Diario El Mercurio denominado "Educación y pandemia" destaca que el ámbito educativo aún permanece una situación de emergencia en donde las políticas adoptadas por el distanciamiento social forzoso, han modificado las condiciones de una educación formal en su infraestructura y estructura comunicativa y se vieron modificadas al acudir a plataformas digitales, en donde la enseñanza se separa de las aulas convencionales y los hogares se transformaron en espacios de trabajo escolar (Negrete, 2020).

Entonces, durante esta modalidad se evidenció aquella verdadera condición de infraestructura y de recursos disponibles especialmente en cada uno de los hogares de estudiantes universitarios tal es el caso presentado en esta investigación de aquellos que pertenecen a la UNACH, ya que provienen de diferentes condiciones sociales y de diferentes regiones y sectores del Ecuador (Cuadro 1).

\section{Estado de la cuestión}

A nivel mundial las universidades no fueron la excepción para trasladar de manera casi inmediata y adoptar la modalidad de clases síncronas mediante plataformas virtuales, un hecho por demás inquietante de conocer si la equivalencia iba dar los mismos resultados en sus estudiantes para que continúen con ese porcentaje de aprendizaje igual o mejor, tal como en la presencialidad, es ahí donde empiezan a surgir muchas interrogantes de investigación globalizada relevantes. Ahora que se pudo experimentar esta fase de transformación digital de la universidad sería un gran error el volver a cometer y afrontar situaciones sin la adecuada reflexión y planificación. Francisco García en su intervención en la IX Conferencia Internacional Digital 2020, destaca que tal vez se evidenció que la Universidad en temas de innovación no está tan madura como se pensaba, lo que invita a tomar en serio el factor estratégico y de liderazgo de los equipos rectorales para construir una universidad digital, inclusiva, participativa y especialmente humana (García-Peñalvo, 2020).

Bajo este enfoque, el primer punto a analizar es el nivel de la llamada "Brecha Digital", un término muchas veces utilizado para definir al carente acceso a la conectividad o el uso de equipos tecnológicos sean estos smartphones, computadoras portátiles o de escritorio; pero como se observa en la Figura 1, se trata de un abismo que crece a medida del tiempo, sobre aquellos aspectos que son notorios en el requerimiento imperioso de combinar las actividades académicas con la tecnología, tal como ocurrió desde marzo del 2020 con las

Licencia Creative Commons Atribución 4.0 Internacional (CC BY 4.0)

Revista Cátedra, 5(1), pp. 47-57, enero-junio 2022. e-ISSN: 2631-2875

https://doi.org/10.29166/catedra.v5i1.3363 
llamadas clases virtuales, que solo fue un encuentro vía internet por parte de profesores y estudiantes mediante videoconferencia. Por lo tanto, referirse a una brecha digital también implica aquellas metodologías innovadoras por parte de los docentes, en tratar de recurrir a patrones pedagógicos en la adaptación de lo presencial a lo estrictamente online, y que al final terminó convirtiéndose en un espacio donde solamente se compartió a los estudiantes publicaciones con contenidos de texto en formato .PDF y videos de la plataforma Youtube.

Brecha de acceso

\section{Brecha de competencias}

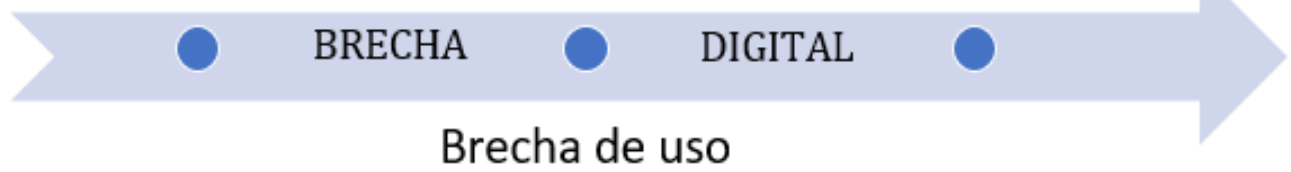

Figura 1. Brecha Digital, adaptado de (García-Peñalvo, 2020)

Si exploramos la realidad de universidades en el mundo, vemos que "este cambio global al aprendizaje en línea sigue el ejemplo establecido por las universidades de China, donde comenzó el brote. Tan rápida adopción global de la educación en línea es asombrosa. Antes del coronavirus, el uso del aprendizaje en línea en la educación superior había mostrado un lento ritmo de cambio" (Abreu, 2020, pág. 2); y ahora se está convirtiendo de manera prolija en la única opción, sobre todo si se repite un confinamiento sean cual sean las razones, por lo tanto, surgen las necesidades de no solamente preguntarse si debe hacerse, sino que tan rápido pueda llevarse a cabo. El rol de la Universidad en el contexto de la pandemia conlleva proyectarse hacia un futuro incierto y donde la carencia de recursos tanto materiales como tecnológicos, buscarán siempre marcar un límite, y donde muchas veces se torna en la excusa de siempre; más bien ahora con esta modalidad en línea las asignaturas deben adecuarse mediante una continua comunicación con los estudiantes y el aprovechamiento de las herramientas tanto síncronas y asíncronas, y a través de una capacidad de respuesta asombrosa, en tiempo récord, se fueron creando espacios de producciones y prácticas en línea para asistir a los docentes; ahí también se fueron dando aquellas oportunidades para el envío de materiales, creación de contenidos, recursos de aprendizaje y de enseñanza, etc.

A partir de ese trabajo mancomunado de autoridades y docentes para construir aquellos espacios equivalentes de lo que existían en la presencialidad y ahora en la virtualidad con miras a continuar con el trabajo académico, surgen otras circunstancias personales en los estudiantes y sus familias, empezando desde reflexionar si cuentan con un ordenador de uso exclusivo y una conectividad propia y de alta velocidad para un correcto seguimiento de la enseñanza virtual (Cabrera, 2020) constata que el nivel formativo de las familias, aunque no de manera exclusiva, actúa como gradiente de la desigualdad (Pérez-López et al., 2021).

Los estudiantes por su lado, en el afán de cumplir con las tareas propuestas, en algunos casos con la guía docente y en otros de manera autónoma ejecutaron sus presentaciones multimedia, sus consultas permanentes mediante la publicación de sus trabajos en blogs y del uso pedagógico que les dieron a las redes sociales como Whatsapp y Facebook. "Los docentes han optado mayoritariamente por un modelo asíncrono acompañado de una interacción docente-discente basada en la comunicación a través de correo electrónico, foros, o chat" (Pérez-López et al., 2021, pág. 339). Se muestra entonces que la comunicación es un factor determinante para evitar el efecto de aislamiento que suele producirse en los modelos de enseñanza a distancia. Casos presentados en países como Estados Unidos mediante la donación de equipos de cómputo y tarjetas de internet a estudiantes de escuelas

Licencia Creative Commons Atribución 4.0 Internacional (CC BY 4.0)

Revista Cátedra, 5(1), pp. 47-57, enero-junio 2022. e-ISSN:2631-2875

https://doi.org/10.29166/catedra.v5i1.3363 
públicas se notó que apenas la mitad de los alumnos en los condados más pobres - y con los mayores porcentajes de indocumentados - estaba asistiendo a las clases en línea. En México, a través de una publicación de diario digital, Nurit Martínez destaca el trabajo de Instituciones de Educación Superior como la Universidad Autónoma Metropolitana (UAM) que entregaron como donación 3,865 tabletas equipadas con tarjetas de internet a alumnos en condición vulnerable de acceso a la tecnología e internet (Martínez, 2020).

Una pequeña parte de la población es beneficiada con estos programas y tienen la posibilidad de que la educación en línea se convierta en la norma para los próximos meses o años, así como también es inmediato el encontrar nuevas soluciones e iniciativas. "Algunos ejemplos son el uso de las plataformas digitales para subir videos y documentos por parte de los docentes y los alumnos, en momentos en que cuenten con internet. Otra opción, que está siendo utilizada por algunas universidades interculturales, es la creación de foros en WhatsApp y Facebook a los que se puede acceder desde los celulares" (Lloyd, 2020, pág. 119).

\section{Metodología}

\subsection{Recolección de datos}

La población que se consideró para esta investigación fueron 9980 estudiantes de las cuatro Facultades (Ingeniería, Ciencias Políticas y Administrativa, Ciencias de la Salud y Ciencias de la Educación) de la UNACH. Esta información tabulada deja constancia que es una realidad nacional puesto que se observa en el Cuadro 1, que los estudiantes de la UNACH pertenecen a las 24 provincias del Ecuador.

El diseño de la investigación es no experimental transeccional descriptivo desde un enfoque cuantitativo de diagnóstico del porcentaje de conectividad, tipo de servicio, en referencia al acceso a dispositivos tecnológicos que tienen los estudiantes universitarios. El enfoque del estudio fue cuantitativo y el alcance de la investigación descriptiva, ya que lo que se buscaba determinar el porcentaje de acceso a las TIC de los estudiantes universitarios en el contexto familiar en tiempos de confinamiento.

\begin{tabular}{lrr}
\hline & № & $\%$ \\
\hline Género & & \\
Femenino & 5640 & 56 \\
Masculino & 4340 & 44 \\
Otro & 0 & 0 \\
Total & 9890 & $100 \%$ \\
Provincia & & \\
AZUAY & 31 & $0,3 \%$ \\
BOLIVAR & 378 & $3,8 \%$ \\
CAÑAR & 131 & $1,3 \%$ \\
CARCHI & 94 & $0,9 \%$ \\
CHIMBORAZO & 5837 & $58,4 \%$ \\
COTOPAXI & 394 & $3,9 \%$ \\
EL ORO & 78 & $0,8 \%$ \\
ESMERALDAS & 67 & $0,7 \%$ \\
GALAPAGOS & 16 & $0,2 \%$ \\
GUAYAS & 58 & $0,6 \%$ \\
IMBABURA & 170 & $1,7 \%$ \\
LOJA & 100 & $1,0 \%$ \\
LOS RIOS & 32 & $0,3 \%$ \\
MANABI & 29 & $0,3 \%$
\end{tabular}




\begin{tabular}{lrr} 
MORONA SANTIAGO & 202 & $2,0 \%$ \\
NAPO & 180 & $1,8 \%$ \\
ORELLANA & 99 & $1,0 \%$ \\
PASTAZA & 214 & $2,1 \%$ \\
PICHINCHA & 503 & $5,0 \%$ \\
SANTA ELENA & 9 & $0,1 \%$ \\
SANTO DOMINGO DE LOS TSACHILAS & 230 & $2,3 \%$ \\
SUCUMBIOS & 126 & $1,3 \%$ \\
TUNGURAHUA & 952 & $9,5 \%$ \\
ZAMORA CHINCHIPE & 50 & $0,5 \%$ \\
TOTAL & 9980 & $100 \%$ \\
\hline
\end{tabular}

Cuadro 1. Recopilación de datos de estudiantes de la UNACH - Género y Provincia de residencia

La recolección de datos se realizó utilizando la técnica de la encuesta y se empleó como instrumento un cuestionario. No se ha seleccionado una muestra, dado que el estudio es descriptivo, por lo que se trabajó con toda la población. Los datos fueron obtenidos en el mes de abril 2020, es decir al inicio del período académico Mayo - Octubre 2020 y para su procesamiento se efectuó un filtrado mediante tablas y hojas de cálculo en Excel, se empleó la estadística descriptiva y se utilizó gráficos de barra para describir cada uno de los resultados obtenidos de cada una de las preguntas del cuestionario aplicado.

\section{Resultados y Discusión}

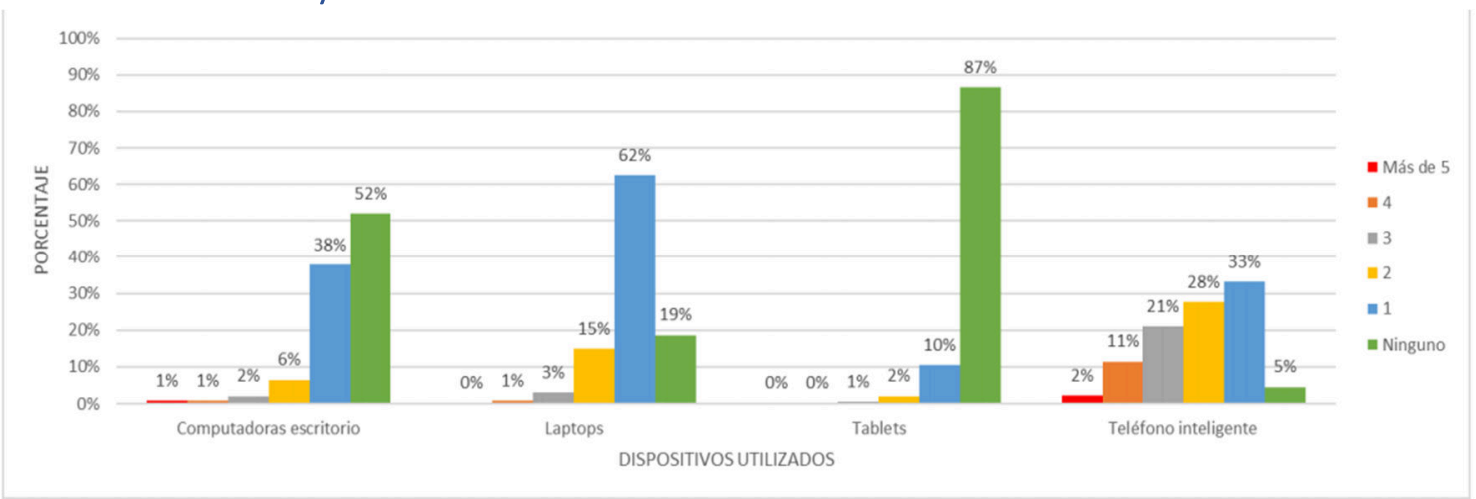

Figura 2. Dispositivos de acceso a estudiantes

Los teléfonos inteligentes entre 1 y 4 dispositivos con un 95\%, las computadoras portátiles (laptops) entre 1 y 2 equipos con un $80 \%$ y las computadoras de escritorio entre 1 y 2 equipos con un $46 \%$ son los porcentajes que los estudiantes de la UNACH hicieron uso para su trabajo académico (Figura 2); se aprecia a simple vista que ha existido variedad de dispositivos y equipos sin embargo, la interpretación es otra, ya que dentro del hogar la realidad ha obligado al intercambio con otros miembros de la familia como se observa en la Figura 3, por lo tanto se analiza que solo un $62 \%$ de computadoras portátiles, un $38 \%$ de computadoras de escritorio y un 33\% de teléfonos inteligentes probablemente fueron exclusivos para realizar dichas actividades.

Es evidente que llevar las clases mediadas por las TIC no ha sido una tarea fácil, especialmente porque ha sido implementada con muy poco tiempo de preparación y antelación. La organización dentro de cada hogar fue un punto fundamental para intercambiar aquellos dispositivos y equipos según las necesidades de cada integrante, y no solamente por el tema académico, sino también por el teletrabajo de sus padres o familiares. Toda esta experiencia vivida, no ha dejado de ser una oportunidad para que tanto docentes como estudiantes exploren las distintas herramientas que las tecnologías de la información

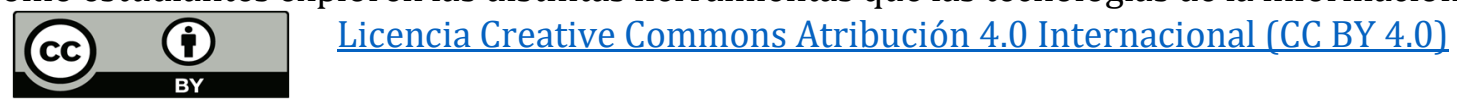


y comunicación pueden ofrecerles. Utilizar el podcast, el YouTube, recursos digitales de editoriales, programación de radio y televisión, guías de autoaprendizaje de modalidades flexibles y así mantener ciertos niveles de interacción, además, de no bajar el nivel de calidad educativa (Chirinos et al., 2020).

Dichos dispositivos debían adaptarse a la consecuente necesidad de actividades que se desarrollaron en las clases tanto síncronas como asíncronas, con la participación foros, o grupos de WhatsApp y Facebook, y por supuesto el acceso desde los teléfonos móviles era indispensable. En la mayoría de los hogares de los estudiantes de la UNACH utilizan entre 4 personas o más la misma conexión a internet dando como consecuencia mediante este dato obtenido que los miembros de la familia se alternan el uso de los dispositivos, y a su vez considerando el anterior análisis en el cual se señalaba que utilizan los dispositivos tanto laptop como computadoras de escritorio (Figura 2). Esto implica un inconveniente muy serio y determinante a la hora de cumplir las tareas síncronas.

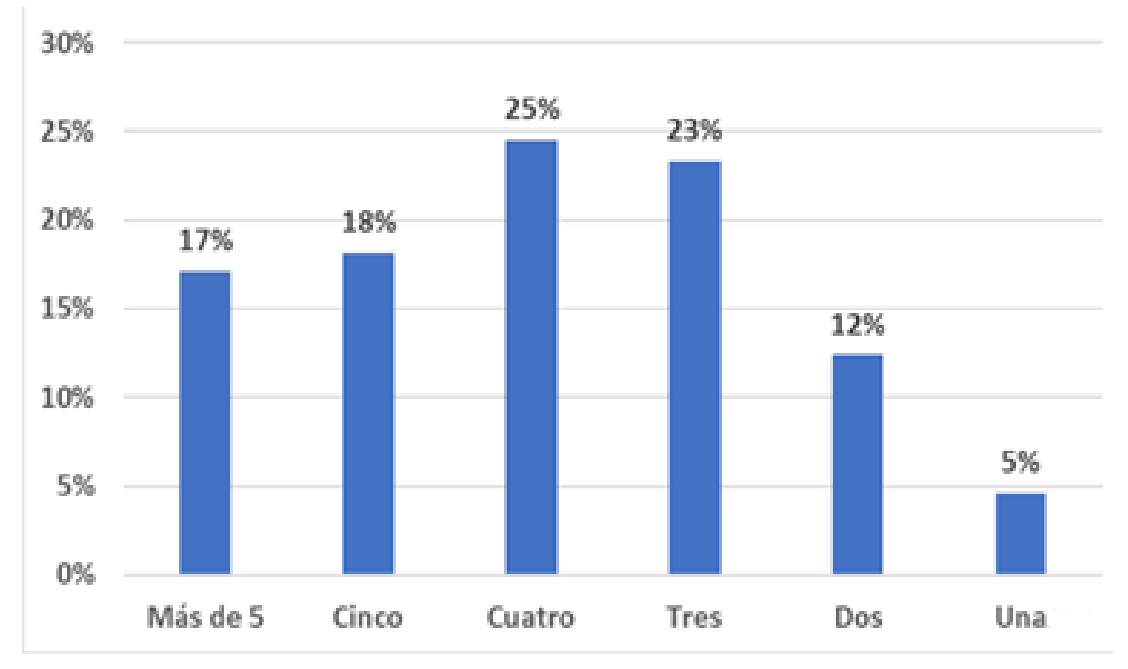

Figura 3. Número de personas que hacen uso del internet en su hogar simultáneamente

Para la mayoría, su primera experiencia con la educación virtual llegó con el confinamiento producto de la pandemia. Asimismo, esta investigación ha abierto otros puntos fundamentales como el indagar acerca de "otras desigualdades que se han agudizado con la crisis sanitaria y económica en el mundo, incluyendo la escasez de dinero y alimentos por el despido en sus trabajos ocasionales; las demandas por cuidar a niños y otros familiares; el aumento en las labores de casa, etc." (Lloyd, 2020, pág. 120). 


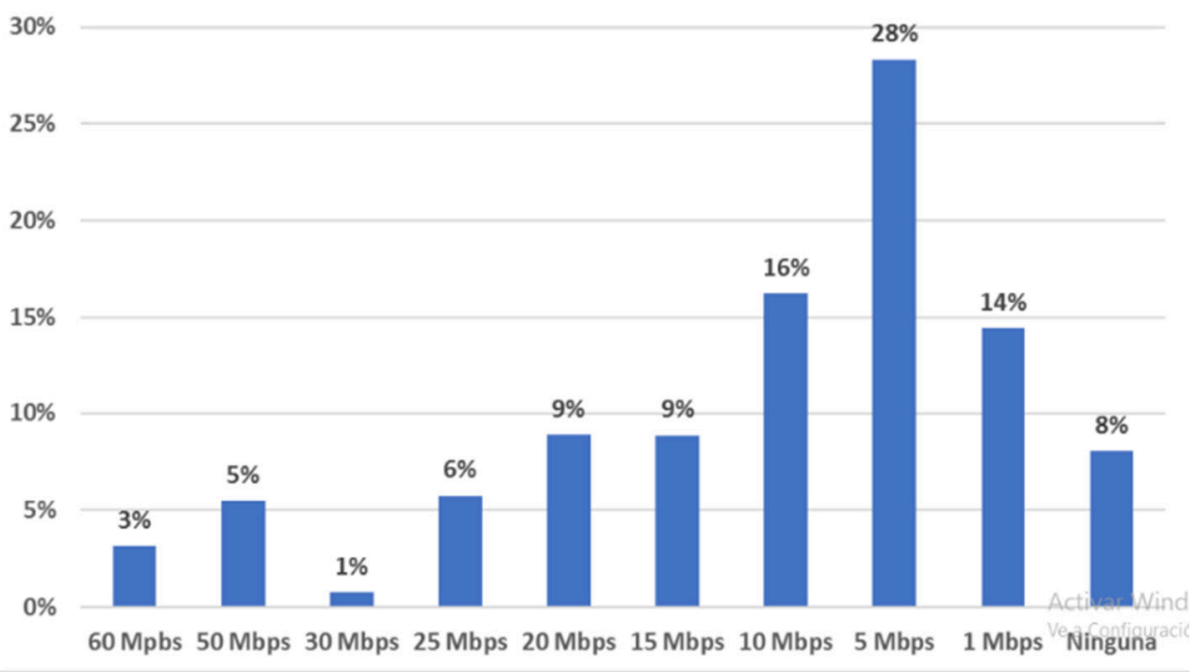

Figura 4. Velocidad del servicio de Internet en los estudiantes

Una vez conocido el porcentaje de la velocidad de internet que posee cada uno de los estudiantes de la Universidad Nacional de Chimborazo se determina que la mayoría no cuenta con un óptimo rendimiento en la conectividad ya que todos los miembros de la familia están ejecutando diferentes actividades de manera simultánea lo que se ve afectado el desarrollo de las tareas y trabajos propuestos.

Tales demandas afectan a quienes tienen menos posibilidades económicas por el costo que implica contratar un servicio de fibra óptica, además dentro de un aspecto poco analizado también está el de las estudiantes mujeres de todos los niveles socioeconómicos; puesto que han asumido una carga mucho mayor de los trabajos en casa durante la contingencia, lo cual necesariamente impacta en su nivel de productividad académica.

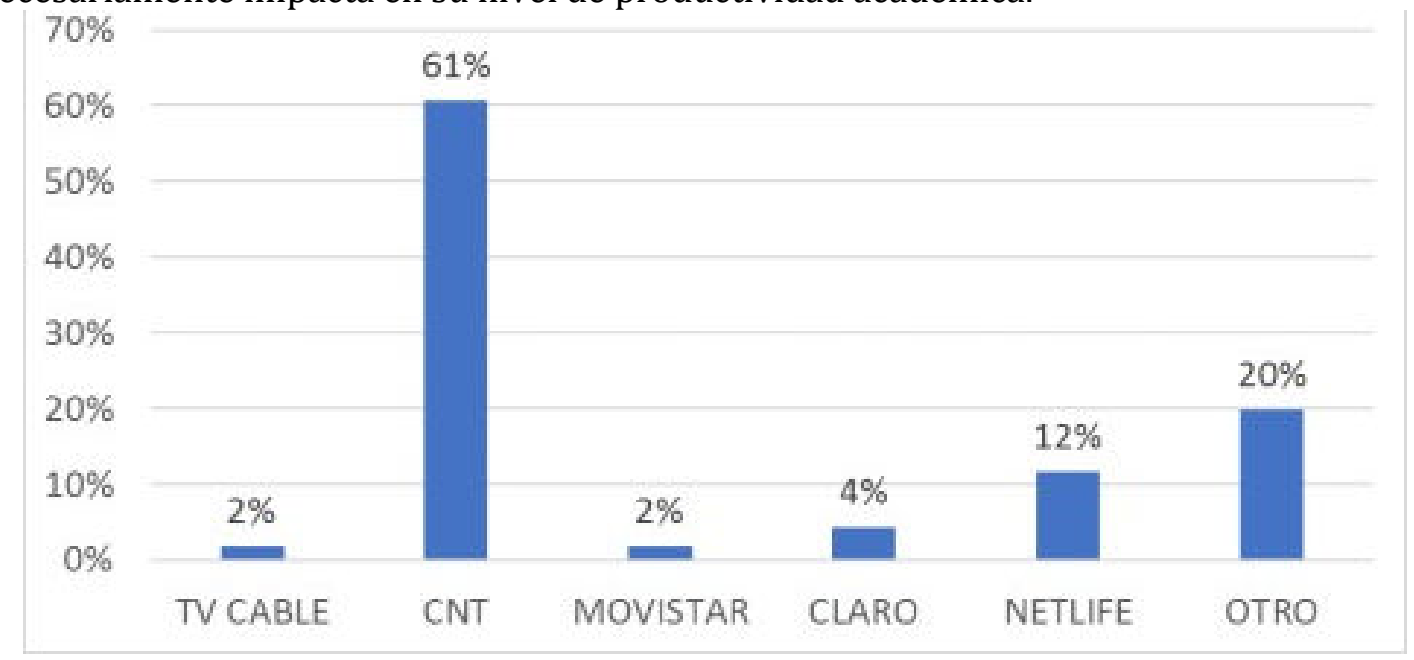

Figura 5. Operadoras para el uso de internet de los estudiantes

Por esta época de confinamiento por la pandemia se aceleró el crecimiento de los servicios de telecomunicaciones, donde el 70,7\% de la población en Ecuador usó Internet, lo que representó 11,5 puntos porcentuales más que en el 2019. En la zona rural creció $14 \%$ y en la urbana, 10,4\%. Del total de usuarios, 92,1\% utilizó Internet una vez al día. También, creció de $59,9 \%$ a $62,9 \%$ el número de teléfonos móviles inteligentes activados (El Comercio, 2021).

En el estudio se observa que los inconvenientes se presentan al contar con un servicio que tiene deficiencias a lo largo de muchos puntos geográficos de la ciudad, provincia y país, ya que muchos estudiantes viven en sectores rurales. Además, también se confirma por medio

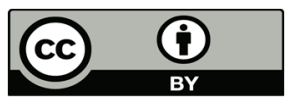

Licencia Creative Commons Atribución 4.0 Internacional (CC BY 4.0)

Revista Cátedra, 5(1), pp. 47-57, enero-junio 2022. e-ISSN:2631-2875

https://doi.org/10.29166/catedra.v5i1.3363 
de este análisis de datos que hay estudiantes que realizan recargas de saldo en sus teléfonos inteligentes para conectarse a las clases síncronas, lo que no es una garantía de eficacia en su aprovechamiento y desarrollo de actividades autónomas en línea.

\section{Conclusiones}

Los datos obtenidos fueron filtrados con base en 3 aspectos importantes como son el tipo de dispositivos, y el número con el que contaban los estudiantes encuestados, lo que al final nos permitió conocer que en un 70\% aproximadamente comparten sus equipos con otros miembros de la familia. Estos datos analizados revelan la realidad de la mayoría de los hogares de los estudiantes universitarios, que durante el tiempo de confinamiento han tenido que buscar formas y maneras para poder cumplir con su trabajo académico. Estas dificultades presentadas como por ejemplo la conexión a internet, obliga a plantearse un tipo de variantes que puedan ayudar y combinar la actividad desarrollada con la educación en línea, y que es propicio para ejecutar otra investigación que determine si fue el factor que muchos estudiantes tomaron al final del semestre como opción de retiro en asignaturas propiamente experimentales.

Al mostrar estas realidades podemos comprobar que los estudiantes no cuentan con dispositivos electrónicos, igualmente no poseen computadoras que les permita trabajar en actividades académicas durante largos periodos de clase síncronas, también se puede observar que un $25 \%$ no ha hecho uso de ningún tipo de dispositivo o equipo (Figura 2) en sus actividades académicas lo cual también es preocupante.

Finalmente, los datos indican que en los hogares se hace muy necesario el internet como un servicio básico, por eso las familias en sus domicilios han requerido la instalación de internet siendo CNT la más solicitada (Figura 5), aunque esta operadora presenta inconvenientes en la calidad del servicio por la intermitencia desde hace mucho tiempo (La Hora, 2021), lo que provoca los continuos abandonos de las sesiones síncronas. 


\section{Bibliografía}

Abreu, J. L. (2020). Tiempos de Coronavirus: La Educación en Línea como Respuesta a la Crisis. Revista Daena (International Journal of Good Conscience), 15(1).

Cabrera, L. (2020). Efectos del coronavirus en el sistema de enseñanza: Aumenta la desigualdad de oportunidades educativas en España. Revista de Sociología de la Educación-RASE, 13(2), 114-139.

Chirinos, M. P., Olivera, N. A. G., \& Cerra, D. C. (2020). En tiempos de coronavirus: Las TIC' S son una buena alternativa para la educación remota. Revista Boletín Redipe, 9(8), 158165.

El Comercio. (2021, mayo 17). El Internet es el servicio que más despuntó el 2020. El Comercio. Recuperado el 10 de septiembre de 2021, de: https://www.elcomercio.com/actualidad/negocios/ecuador-internet-tecnologiafibra-optica.html

García-Peñalvo, F. J. (2020). E-Learning en tiempos de COVID-19.

La Hora. (2021, julio 29). CNT, una empresa supuestamente rentable y con problemas. Recuperado el 10 de septiembre de 2021, de: https://www.lahora.com.ec/pais/cntproblemas-antiguos/

Lloyd, M. (2020). Desigualdades educativas y la brecha digital en tiempos de COVID-19.

Martínez, N. (2020). UAM propone entregar más de 3 mil tabletas a alumnos en condición vulnerable. El Sol de México / Noticias, Deportes, Gossip, Columnas. Recuperado el 10 de septiembre del 2021, de:https://www.elsoldemexico.com.mx/mexico/uampropone-entregar-mas-de-3-mil-tabletas-a-alumnos-en-condicion-vulnerable5116063.html

Negrete, N. (2020). Educación y pandemia / Diario El Mercurio. Recuperado el 10 de septiembre de 2021, de: https://elmercurio.com.ec/2020/07/02/educacion-ypandemia/

Pérez-López, E., Atochero, A. V., \& Rivero, S. C. (2021). Educación a distancia en tiempos de COVID-19: Análisis desde la perspectiva de los estudiantes universitarios. RIED. Revista Iberoamericana de Educación a Distancia, 24(1), 331-350.

\section{Autores}

JORGE SILVA-CASTILLO obtuvo su título de Maestría en Informática Educativa en la Escuela Superior Politécnica de Chimborazo (Ecuador) en 2016. Obtuvo el título de Licenciado en Ciencias de la Educación Profesor de Informática Aplicada a la Educación en la Universidad Nacional de Chimborazo en 2007.

Actualmente es profesor ocasional de La Carrera de Pedagogía de las Ciencias Experimentales Informática de la UNACH. Es Coordinador del Programa de Maestría en Educación, mención Tecnología e Innovación Educativa del Instituto de Posgrado de la Universidad Nacional de Chimborazo. Es Investigador Adjunto del Grupo de Investigación UMAYUK de las TIC aplicadas a la Educación - UNACH (Ecuador). Sus principales temas de investigación incluyen los PLE, los EVA, la Digiculturalidad, los Objetos de Aprendizaje. Es autor y coautor de varios capítulos de libro y artículos publicados en varias conferencias (US, UH, UNACH, CEDIA) y revistas regionales (Latindex) de alto impacto (Scopus).

MIRIAN PEÑAFIEL-RODRIGUEZ obtuvo su título de Maestría en Gerencia Educativa en la Universidad Estatal de Bolívar (Ecuador) en 2006, y Maestría en Pedagogía mención Docencia Intercultural en la Universidad Nacional de Chimborazo (Ecuador) en 2021. Obtuvo el título de Licenciada en Ciencias de la Educación especialidad Filosofía y Ciencias Socio económicas en la Universidad Nacional de Chimborazo (Ecuador) en 2002. 
Actualmente es profesora ocasional en la Carrera de Educación Inicial de la Facultad de Ciencias de la Educción, Humanas y Tecnologías de la Universidad Nacional de Chimborazo. Es investigadora adjunta en varios proyectos de la Facultad de Ciencias de la Educación de la UNACH. Sus principales temas de investigación incluyen: Interculturalidad, Estrategias metodológicas de Aprendizaje, Metodologías activas, Actualización del Marco normativo de las Necesidades Educativas Especiales (NEE). Es autora de capítulos de libros y artículos publicados en revistas regionales (Latindex, Redalcy, Scielo).

AMPARO CAZORLA-BASANTES obtuvo su título de PhD en Historia de la Universidad Católica de Valparaiso(Chile) en 2017. Obtuvo el título de Maestría en Gestión Académica Universitaria de la Universidad Nacional de Chimborazo (Ecuador) en 2005. Obtuvo el título de Doctora en Ciencias de la Educación mención Pedagogía y Gerencia Educativa de la Universidad Nacional de Chimborazo en 2000. Obtuvo el título de Licenciada en Ciencias de la Educación, en la especialización de Historia y Geografía de la Universidad Nacional de Chimborazo en 1997.

Actualmente es Decana y profesora titular de la Facultad de Ciencias de la Educción, Humanas y Tecnologías de la Universidad Nacional de Chimborazo. Es articulista y miembro del Consejo de la Revista Chakiñan (Ecuador). Es Docente Investigadora en el Área de Historia. Sus principales temas de investigación incluyen: La transformación cultural ideológica de la sociedad quiteña. Las representaciones socioculturales en la formación docente de la Universidad Nacional de Chimborazo. Influencia de la antigüedad y del total de las horas de investigación en la producción científica de docentes universitarios. (2018). Es autora de Libros, capítulos de libros y artículos publicados en revistas de alto impacto (Scopus, Latindex, Redalcy, Scielo). 